Design Observational cohort study

Setting British Olympic network

Patients (or Participants) Men's and Women's Great Britain boxing, skeleton and hockey and elite women's rugby

Interventions (or Assessment of Risk Factors) Baseline SMBA and Y-balance measures were taken as part of a bi-annual squad profiling battery.

Concussion was diagnosed by one of the team physiotherapists or sports medicine consultants. Testing was repeated within three days of initial injury (where possible) and upon clearance for return to play following completion of the return-to-play protocol.

Main Outcome Measurements Postural sway (SMBA) and the Y-balance test

Results To date five concussions have been recorded across all groups, demonstrating a mean percentage reduction in postural sway of $18.3 \%$ (87.2 SD $=11.3-71.2 \mathrm{SD}=23.7)$. Ther was no significant difference in baseline postural sway between the concussed and non-concussed groups $(p=0.204)$.

For Y-balance, a $0.6 \%$ drop in return to play (RTP) reach was recorded in the concussed group when compared to group baseline scores $(\mathrm{p}=0.852$, mean baseline- $96.3 \mathrm{~cm} \mathrm{SD}=$ 2.3 , RTP $95.7 \mathrm{~cm} \mathrm{SD}=3.94)$.

Conclusions This study highlights a trend towards a residual reduction in somatosensory function upon return to play following concussion, despite normalisation of symptoms and SCAT5 testing. Despite this not being a statistically significant change, the study offers a promising start to warrant further investigation of somatosensory function and its relationship with increased MSK injury rates.

\section{THE RELATIONSHIP BETWEEN NECK STRENGTH AND CONCUSSION IN MALE PROFESSIONAL RUGBY PLAYERS}

1,2Theo Farley, 'Ed Barry, ${ }^{1}$ Richard Sylvester, 'Akbar De Medici, 'Mathew Wilson. ${ }^{1} T$ The Institute of Sport Exercise and Health, London, UK; ${ }^{2}$ The English Institute of Sports, London, UK

\subsection{6/bjsports-2021-IOC.372}

Background Sports related concussion has become one of the highest burden injuries within professional and amateur sports. There is currently limited evidence as to the existence of modifiable intrinsic risk factors to concussion however lab and field based studies indicate that muscle function may be a significant factor in reducing an external force and therefore the head accelerations thought to lead to a concussive injury. To date only one study supports the association of neck strength and concussion incidence with no unique range known to be significant.

Objective To investigate whether neck strength is significantly associated with concussion incidence in a group of male professional rugby players.

Design Prospective cohort study

Setting Conducted in a National Rugby base on 225 elite rugby union club players, during the 2018-2019 season.

Interventions Athletes were assessed for neck strength at three time points throughout the season using a method of selfresisted isometric contraction.

Main Outcome Measurements Associations with diagnosed concussion injuries are presented as Incidence Rate Ratios (IRR) with 95\% confidence intervals. we present the IRR for a $10 \%$ increase in each variableand compared results against concussion using match minutes to allow for risk exposure.
Results A $10 \%$ increase in extension strength was associated with a $13 \%$ decrease in concussion risk.

A neck extension strength score of $41.2 \mathrm{~kg}$ or below indicated athletes most at risk of sustaining a concussion with a true positive rate of $71.4 \%$ and a false positive rate of $46.1 \%$ Conclusions To our knowledge this is the first study to demonstrate a unique strength range associated with concussion and to identify a minimum strength score associated with a quantifiable risk. Neck extension strength is a modifiable intrinsic risk factor to concussion in professional male rugby players and may be considered as part of a pre-season strength and conditioning regime.

\section{DOES A MANDATORY 36-T0-48-HOUR LATER ASSESSMENT FOR SPORT-RELATED CONCUSSIONS REDUCE THE RISK OF ATHLETES INAPPROPRIATELY RETURNING TO PLAY?}

\footnotetext{
1,2Steffan Griffin, ${ }^{6}$ Matt Cross, ${ }^{1}$ Lewis Henderson, ${ }^{4}$ Geraint Ashton Jones, ${ }^{1,3}$ Keith Stokes, ${ }^{1,5}$ Simon Kemp. ${ }^{1}$ Rugby Football Union, London, UK; ${ }^{2}$ University of Edinburgh, Edinburgh, UK; ${ }^{3}$ University of Bath, Bath, UK; ${ }^{4}$ Alligin Performance, Glasgow, UK; ${ }^{5}$ London School of Hygiene and Tropical Medicine, London, UK; ${ }^{6}$ Premiership Rugby Limited, London, UK
}

\subsection{6/bjsports-2021-IOC.373}

Background Concussion is one of the key player welfare issues in rugby union. A post-match assessment is a mandatory part of the head injury assessment (HIA) process in elite men's rugby union, something unique to the sport and not seen in many other sporting settings. There is little evidence surrounding the potential value of this mandatory post-match assessment.

Objective To analyse at what stage of rugby union's head injury assessment (HIA) protocol match-related concussions are diagnosed over the course of one season.

Design Prospective analysis.

Setting Twelve professional rugby union clubs in the highest league in England (UK) in 2019-2020.

Participants Professional male rugby union players who were diagnosed with a match-related concussion across all competitions.

Outcome measures Number and proportion of concussions diagnosed at different stages of HIA protocol: HIA1 (ingame, using an abridged version of the Sports Concussion Assessment Tool 5 (SCAT5); HIA2 (within 3-hours of match, supported by the SCAT5); HIA3 (within 48-hours of match, supported by the SCAT5); or outside of these windows.

Main Results There were 109 concussions (incidence 21.3/ 1000 player hours) across all competitions. 32 (29\%) of concussions were formally diagnosed in game at the HIA1 stage. $65(60 \%)$ of concussions were formally diagnosed at the HIA2 stage, and $9(8 \%)$ post-match at the HIA3 stage. Three (3\%) were diagnosed outside of the HIA window.

Conclusions The majority of formal diagnoses were made on match-day (in-game or post-match). However, the number of diagnoses made outside of this time period suggests that a later mandatory formal clinical assessment, based on the widely available SCAT5, can help clinicians to identify delayed presentations. As such, later assessments could help minimise the risk of players inappropriately returning to sport without following the appropriate graduated return-toplay protocol. 